\title{
Experimental Research on Electric Energy Consumption and Control Method of Electric Vehicle
}

\author{
Lifu $\mathrm{Li}^{\mathrm{a}}$, Wanli Dong ${ }^{\mathrm{b}}$ \\ School of Mechanical \& Automotive Engineering, South China University of Technology, \\ Guangdong Key Laboratory of Automotive Engineering, Guangzhou 510641, P.R China \\ aemail: pmlfli@scut.edu.cn, bemail:627803820@qq.com
}

Keywords: electric vehicle; energy consumption experiment; driving cycle; control method

\begin{abstract}
As the electric energy consumption of pure electrical vehicle(EV) is huge, the driving range of $\mathrm{EV}$ is usually short. To solve this problem, the energy consumption experiment of EV was carried out with the electric energy consumption testing system in NEDC and Jap1015 driving cycles. Then the effect of electric energy consumption on the entire driving cycle during different variable speed stages was investigated. Also, the relationship between the electric energy consumption and the dynamic control method of electric energy under NEDC and Jap1015 driving cycle was analyzed. Results show that the energy consumption of the entire driving cycles depends on the accelerating stages, and the electric energy consumption of EV does not increase as the driving distance adds up in different driving cycles. The dynamic control method of electric energy is the defining factor of electric energy consumption. When the three accelerating stages of NEDC former part are controlled with dynamic control method based on instantaneous energy consumption per kilometer, the energy consumption per kilometer will decrease effectively.
\end{abstract}

\section{Introduction}

With the advantages of zero emission, low noise and driving economy, EVs are brought into sharp focus. However, the huge energy consumption and short driving range severely restrict the development of EV. Therefore, increasing the driving range of EV through reducing the electric energy consumption has become one of the key research directions in the field of EV ${ }^{[1]}$. In recent years, researchers have made great researches on the electric energy consumption of $\mathrm{EV}$. The relationship between vehicle parameters and electric energy consumption of EV was analyzed in [2][3]. [4] discussed the evaluation system of EV energy consumption and suggested that energy usage ratio can be used as a synthetic evaluation indicator of vehicle tractive performance and economy. Influence on the electric energy consumption of EV caused by different drivers, acceleration, speed and braking energy recovery were analysed in [5].[6] studied the testing method of electric energy consumption of $\mathrm{EV}$, and tested the energy consumption of $\mathrm{EV}$ at different temperatures. [7] estimated the energy consumption of EV by combining the GPS system with simulation software of vehicle power system.

However, in current studies, the influence of energy consumption dynamic change and dynamic control method of electric energy on the energy consumption of EV are less considered. According to vehicle theory ${ }^{[9]}$, under certain load conditions and driving cycles, the electric energy consumption of EV depends upon the corresponding control method of electric energy of different speed and acceleration. This paper uses a certain type of EV as the study object and analyses the relationship between dynamic change of electric energy consumption and different driving cycles, dynamic control method of electric energy, which can provide technical support for developing the control strategies and methods based on electric energy consumption and thus decreasing the electric energy consumption.

\section{The Energy Consumption Experiment of EV}

To analyze the relationship between dynamic control methods of batteries output current and electric energy consumption under different driving cycles, using a certain type of EV as the study 
object. The main technical parameters are shown in Table 1. The electric energy consumption testing system was used to test the energy consumption of EV in NEDC and Jap1015 driving cycles.

Table 1 The Main Technical Parameters of EV

\begin{tabular}{cc}
\hline Parameters & Value \\
\hline Curb Weight/ kg & 2380 \\
Maximum Speed/ $(\mathrm{km} / \mathrm{h})$ & $\geq 140$ \\
Peak Power of Motor/ kW & 90 \\
Max Torque of Motor/ Nm & 450 \\
Ratio of Transmission System & 6.42 \\
Tyre Specifications & $225 / 65 \mathrm{R} 17$ \\
\hline
\end{tabular}

And the electric energy consumption testing system consists of AVL Roadsim chassis dynamometer and HIOKI 3390 electric energy tester, Fig 1 is the physical map of the system.

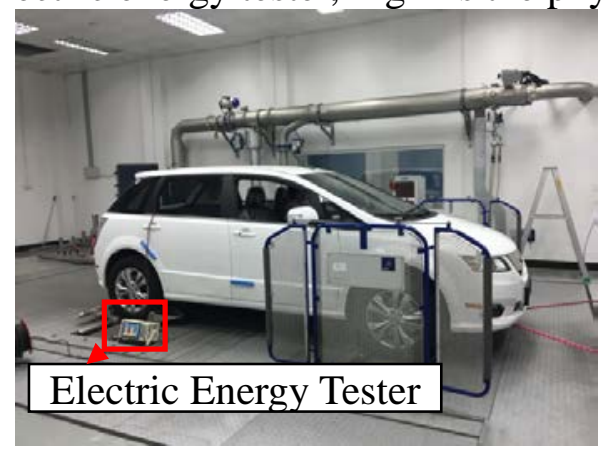

Fig 1 Electric Energy Consumption Testing System of EV

As shown in Fig.1, the structure of the testing system and the principles of this system are as follows, put EV on the chassis dynamometer and keep the car running when experimenting. The chassis dynamometer loads on the driving wheels according to NEDC, Jap1015 time-speed curves, the rotate speed of chassis dynamometer dyno is measured by revolution speed transducer. Then the vehicle speed is calculated according to the radius and rotate speed of the dyno, and the acceleration can be calculated through deriving the vehicle speed. Meanwhile, the data of battery output energy is measured by the electric energy tester.

The experiment was carried out according to the regulations of Chinese standard GB 18386-2005, the energy regeneration and the energy consumption of accessories were not considered. Before the experiment, adjusted the pure electric vehicle and chassis dynamometer relative position, and the electric energy tester and the EV power battery connected. During the testing process, the driver operated the accelerator pedal to ensure that the actual speed transformation followed the change of NEDC and Jap1015 driving cycles. Meanwhile, the data of vehicle $u_{i}(t)$, battery output current $I_{\text {ess. } i}(t)$, voltage $U_{\text {ess. } i}(t)$ and power $P_{\text {ess. } i}(t)$ during $0 \sim 617$ s (experimental time for short) were recorded with electric energy consumption testing system. And $i=1,2$ refers to the NEDC, Jap1015 driving cycles respectively. Fig. 2 (a) and (b) are the curves of $u_{i}(t)$-time.

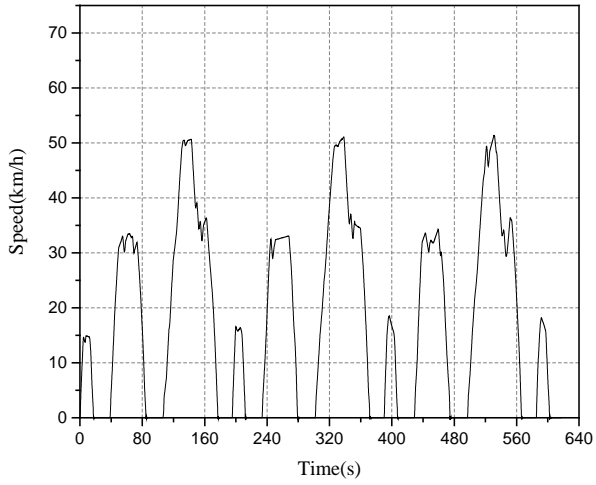

(a)NEDC driving cycle

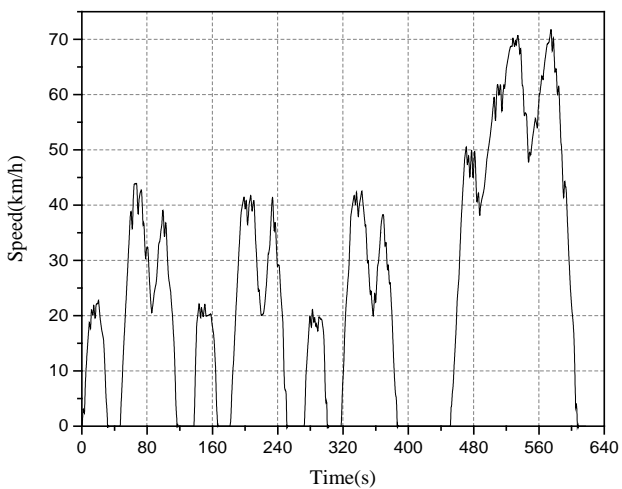

(b)Jap1015 driving cycle

Fig. 2 the curve of $u_{i}(t)$-time about NEDC Jap1015 driving cycles 


\section{The Analysis of Energy Consumption Experiment of EV}

The energy consumption per kilometer can be used as an indicator of energy economy of $\mathrm{EV}^{[4]}$.When EV follows certain driving cycle, the instantaneous energy consumption per kilometer $E_{\text {cr.i }}(t)$ during ith driving section $\left[t_{i} \sim t_{i+1}\right](i=1,2, \cdots n)$ can be expressed as:

$E_{\text {cr. } i}(t)=\frac{\int_{t_{i}}^{t_{i+1}} k_{1} u_{i}(t)\left[k_{2}+k_{3}\left(u_{i}(t)\right)^{2}+\delta m a_{i}(t)\right]+B d t}{\int_{t_{i}}^{t_{i+1}} u_{i}(t) d t}$.

Where $k_{1}=1 / 3.6 \eta_{\mathrm{T}} \eta_{\mathrm{m}} \eta_{\mathrm{V}} ; k_{2}=m g(f \cos \alpha+\sin \alpha) ; k_{3}=C_{\mathrm{D}} A / 21.15 ; B=P_{\mathrm{ac}} / 3600 . m$ is the curb weight in kg; $f$ is the rolling resistance coefficient; $\alpha$ is the grade of the road; $C_{\mathrm{D}}$ is the aerodynamic drag; $A$ is the vehicle frontal area in $m^{2} ; u_{i}(t)$ is the speed in $\mathrm{km} / \mathrm{h} ; a_{i}(t)$ is acceleration in $\mathrm{m} / \mathrm{s}^{2} ; \delta$ is coefficient of the revolving. The average energy consumption per kilometer $\overline{E_{\text {cr }}}$ can be expressed as:

$$
\overline{E_{\mathrm{cr}}}=\frac{\sum_{i=1}^{n}\left\{\int_{t_{i}}^{t_{i+1}} k_{1} u_{i}(t)\left[k_{2}+k_{3}\left(u_{i}(t)\right)^{2}+\delta m a_{i}(t)\right]+B d t\right\}}{\sum_{i=1}^{n} \int_{t_{i}}^{t_{i+1}} u_{i}(t) d t} .
$$

Based on the working principle of EV, the mileage $s_{i}$, electric energy consumption $W_{i}$ and average energy consumption per kilometer $\overline{E_{\text {cr. } i}}$ can be calculated in NEDC and Jap015 driving cycles during experimental time, as shown in Table 2.

Table 2 Experimental data of EV in two driving cycles

\begin{tabular}{ccc}
\hline Parameters & $\begin{array}{c}\text { NEDC } \\
\text { driving cycles }\end{array}$ & $\begin{array}{c}\text { Jap1015 driving } \\
\text { cycles }\end{array}$ \\
\hline Mileage $/ \mathrm{km}$ & 3.06 & 4.19 \\
Electric energy consumption $/ \mathrm{Wh}$ & 664.05 & 602.37 \\
Average energy consumption per & 216.87 & 143.77 \\
kilometer $/(\mathrm{Wh} / \mathrm{km})$ & & \\
\hline
\end{tabular}

In Table 2, when $S_{2}>S_{1}, W_{2}<W_{1}$, that the mileage $S_{2}$ in Jap1015 driving cycle is higher than that of NEDC cycle. But the electric energy consumption of Jap1015 driving cycle is lower than NEDC. Therefore, it can be concluded that the electric energy consumption $W_{i}$ isn't positively related to mileage $S_{i}$ in different driving cycles. NEDC and Jap1015 driving cycles normally consist of two parts which include the former and the later part. The former part is made up of cycles of $m(m=123)$ units, and each unit contains $\mathrm{j}(\mathrm{j}=\mathrm{a}$ u s stand for acceleration uniform and deceleration) different speed changing stages. To study the effect of control methods $M_{k}(k=1,2,3)$ of battery output current on the electric energy consumption of EV in acceleration, deceleration and uniform stages in different driving cycles, the mileage $S_{i}^{j k m}(t)$, electric energy consumption $W_{i}^{j k m}$ and average energy consumption per kilometer $\overline{E_{\text {cr.i }}^{j k m}}$ were calculated, as is showed in Table 3.

As is showed in Table 3, in the two driving cycles, $\overline{E_{\mathrm{cr} . i}^{a 11}}>\overline{E_{\mathrm{cr} . i}^{u 11}}>\overline{E_{\mathrm{cr} . i}^{s 11}}, W_{i}^{a 11}>W_{i}^{u 11}>W_{i}^{s 11}$, and accelerating stage $W_{i}^{a 11}$ account for $61.94 \%, 65.61 \%$ of two driving cycles $W_{i}$ respectively. Therefore, it can be concluded that the electric energy consumption $\overline{E_{\text {cr. } i}}$ of the entire driving cycle depends on the accelerating stage $\overline{E_{\mathrm{cr} . i}^{a k m}}$. 
Table 3 The experimental data of PEB in different speed changing stages of two driving cycles

\begin{tabular}{c|cc|cc|cc}
\hline Parameters & \multicolumn{2}{|c|}{ Acceleration $(\mathrm{j}=\mathrm{a})$} & \multicolumn{2}{c|}{ Uniform $(\mathrm{j}=\mathrm{u})$} & \multicolumn{2}{c}{ Deceleration $(\mathrm{j}=\mathrm{d})$} \\
\hline $\begin{array}{c}\text { Driving cycles } \\
\text { Mileage / } S_{i}^{j 11} \mathrm{~km}\end{array}$ & NEDC & Jap1015 & NEDC & Jap1015 & NEDC & Jap1015 \\
$\begin{array}{c}\text { Electric energy consumption } \\
W_{i}^{j 11} / \mathrm{Wh}\end{array}$ & 409.57 & 395.16 & 234.28 & 150.86 & 17.38 & 56.30 \\
$\begin{array}{c}\text { Average energy consumption } \\
\text { per kilometer }\end{array}$ & 539.49 & 237.06 & 137.40 & 120.61 & 29.11 & 44.26 \\
$\frac{E_{c r, i}^{j 11} /(\mathrm{Wh} / \mathrm{km})}{}$ & & & & & & \\
\hline
\end{tabular}

According to the principle of energy conversion of $\mathrm{EV}$, when the car is running, the battery takes the constant voltage discharge pattern, in which different control methods $\mathrm{M}_{\mathrm{k}}$ are applied to regulate battery output current $I_{\text {ess }}(t)$ so that the motor can get the electric energy it needs to adapt to the changing road conditions. To discuss the relationship between $\mathbf{M}_{k}$ and $\overline{E_{\text {cr.i }}^{a k m}}$, taking the a1 accelerating stage of NEDC as the study project, and conducted the electric energy consumption experiment with $\mathrm{M}_{k}$ methods. The speed $u_{1}^{a k 1}(t)$, instantaneous energy consumption per kilometer $E_{\text {cr.1 }}^{a k 1}(t)$ and battery output current $I_{\text {ess.1 }}^{a k 1}(t)$ were got in Fig.3(a), (b), (c).

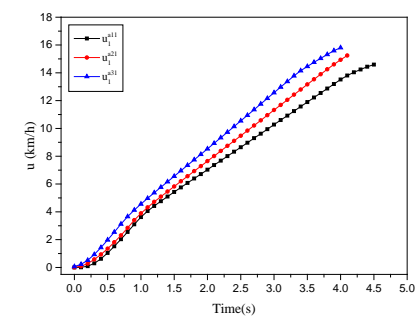

(a)Speed- time curve

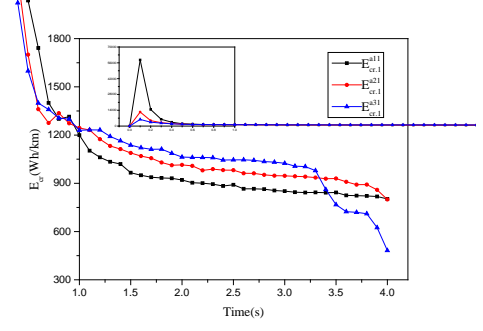

(b) Instantaneous energy consumption per kilometer time curve

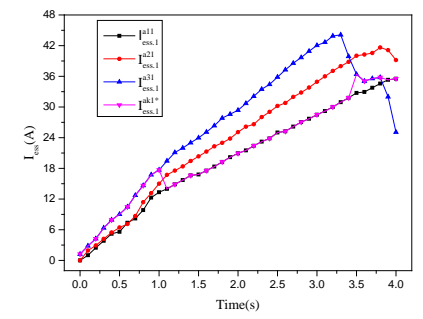

(c) Battery output current -time curve

Fig 3 The experimental data-curve of the accelerate stage on $\mathrm{m}=1$ unit with $\mathbf{M}_{k}$ methods

To describe $u_{1}^{a k 1}(t)$ varying with time, regression analyses were done upon curves of Fig. 3 (a), the expression of $u_{1}^{a 11}(t), u_{1}^{a 21}(t), u_{1}^{a 31}(t)$ were calculated in (3).

$$
\left\{\begin{array}{l}
u_{1}^{a 11}(t)=-0.60+4.15 t-0.16 t^{2}-0.002 t^{3} \\
u_{1}^{a 21}(t)=-0.50+4.42 t-0.20 t^{2}+0.015 t^{3} \\
u_{1}^{a 31}(t)=-0.23+4.75 t-0.15 t^{2}-0.0062 t^{3}
\end{array} .\right.
$$

It can be seen from formula (3), when $t=4 \mathrm{~s}, \quad u_{1}^{a 11}(4)=13.51$, $u_{1}^{a 21}(4)=14.94, u_{1}^{a 31}(4)=15.81 \mathrm{~km} / \mathrm{h}$, that $u_{1}^{a 21}(4)$ basically equals given speed $u_{1}(4)_{e}(15$ $\mathrm{km} / \mathrm{h}$ ), however, $u_{1}^{a 11}(4)<u_{1}(4)_{e}$, it lag behind given time about $0.64 \mathrm{~s}$; but $u_{1}^{a 31}(4)>u_{1}(4)_{e}$, the overshoot is $5.40 \%$.

It can be seen from Fig. 3(b), when $0 \leq t \leq 4.0 \mathrm{~s}, \overline{E_{\mathrm{cr} .1}^{a 21}}$ is maximum, and $\overline{E_{\mathrm{cr} .1}^{a 11}}$ is minimum. Similarly, regression analysis were done upon curves of Fig. 3 (c), and the expression of $I_{\text {ess. } 1}^{a k 1}(t)$ were calculated as equation (4).

$$
\left\{\begin{array}{l}
I_{\text {ess.1 }}^{a 11}(t)=-0.169+12.18 t+3.83 t^{2}-4.79 t^{3}+1.57 t^{4}-0.167 t^{5} \\
I_{\text {ess. } 1}^{a 21}(t)=0.60+6.54 t+17.28 t^{2}-13.85 t^{3}+4.21 t^{4}-0.44 t^{5} \\
I_{\text {ess. } 1}^{a 31}(t)=0.74+19.21 t-1.10 t^{2}-2.83 t^{3}+1.68 t^{4}-0.27 t^{5}
\end{array} .\right.
$$


It can be seen from formula (4) and Fig. 3(b) (c), when the car starts (before 1s), $I_{\text {ess. } 1}^{a 11}<I_{\text {ess. } 1}^{a 21}<I_{\text {ess. } 1}^{a 31}$ and $E_{\text {cr. } 1}^{a 11}>E_{\text {cr. } 1}^{a 21}>E_{\text {cr. } 1}^{a 31}$ and this demonstrates that $E_{\text {cr. } 1}^{a k 1}(t)$ decreases as $I_{\text {ess.1 }}^{a k 1}(t)$ increases during the process; When the car is accelerating (1 3.3s), if definite $K_{\text {ess. } 1}^{a k m}(\mathrm{t})=\mathrm{d}\left[I_{\text {ess. } 1}^{a k m}(t)\right] / d t$, when $\quad K_{\text {ess. } 1}^{a 11}(t)<K_{\text {ess. } 1}^{a 21}(t)<K_{\text {ess.1 }}^{a 31}(t), \quad E_{\text {cr. } 1}^{a 11}(t)<E_{\text {cr.1 }}^{a 21}(t)<E_{\text {cr.1 }}^{a 31}(t)$, which demonstrates that $E_{\text {cr.1 }}^{a k 1}(t)$ increases as $I_{\text {ess.1 }}^{a k 1}(t)$ increases during accelerating process; At the end of acceleration process (3.3 4.0s), $K_{\text {ess.1 }}^{a 31}$ is much less than $K_{\text {ess.1 }}^{a 21}$ and $K_{\text {ess.1 }}^{a 11}$, meanwhile $E_{\text {cr.1 }}^{a 31}$ descends to the lowest value rapidly. Thus different control methods $M_{k}$ of battery output current can directly affect instantaneous energy consumption per kilometer $E_{\text {cr.1 }}^{a k 1}$ of EV. As adjust a1 (j=a, $\mathrm{m}=1$ ) accelerate stage of NEDC, with the $I_{\text {ess.1 }}^{a k m}(t)$ control method $\mathrm{M}_{k}^{* 1}$ which is designed based on instantaneous energy consumption per kilometer, the formula of $I_{\text {ess.1 }}^{a k 1^{*}}(t)$ can be described as equation (5).

$$
I_{\text {ess. } 1}^{a k *^{*}}(t)=0.46+25.16 t-14.34 t^{2}+3.70 t^{3}-0.02 t^{4}-0.07 t^{5} \text {. }
$$

The curve of $I_{\text {ess.1 }}^{a k *^{*}}(t)$ see Fig. 3(c), and it can be concluded that during $4 \mathrm{~s}, \overline{E_{\mathrm{cr} .1}^{a k 1^{*}}}$ equals 894.59Wh/km which is $1.7 \%$ less than $\overline{E_{\text {cr.1 }}^{a 11}}$, 9.4\% less than $\overline{E_{\text {cr.1 }}^{a 21}}$ and $8.3 \%$ less than $\overline{E_{\text {cr.1 }}^{a 31}}$. Similarly, adjusting the $I_{\text {ess. } 1}^{a k m}(t)$ in the a2 a3 accelerating stages of NEDC and designing $\mathrm{M}_{k}^{* 2}$ and $\mathrm{M}_{k}^{* 3}$, which is designed with the same rule as $\mathrm{M}_{k}^{* 1}$. The formula of battery output current can be described in (6) (7).

$$
\begin{aligned}
& I_{\text {ess. } 1}^{a k 2^{*}}(t)=1.78+7.24 t-1.21 t^{2}+0.37 t^{3}-0.03 t^{4}+0.0005 t^{5} . \\
& I_{\text {ess. } 1}^{a k 3^{*}}(t)=1.79+1.93 t+0.85 t^{2}-0.15 t^{3}+0.009 t^{4}-0.00017 t^{5} .
\end{aligned}
$$

When the EV is controlled following the $I_{\text {ess. } 1}^{a k 2^{*}}(t)$, it turned out that during $12 \mathrm{~s}, \overline{E_{\text {cr.1 }}^{a k 2^{*}}}$ equals $505.44 \mathrm{Wh} / \mathrm{km} 15.05 \%$ less than $\overline{E_{\mathrm{cr} .1}^{a 12}}, 18.61 \%$ less than $\overline{E_{\mathrm{cr} .1}^{a 22}}$ and $17.71 \%$ less than $\overline{E_{\mathrm{cr} .1}^{a 32}}$. When the $\mathrm{EV}$ is controlled following the $I_{\text {ess. } 1}^{a k 3}(t)$, during $26 \mathrm{~s} \overline{E_{\text {cr. } 1}^{a k 3^{*}}}$ equals $395.40 \mathrm{Wh} / \mathrm{km}, 19.19 \%$ less than $\overline{E_{\text {cr.1 }}^{a 13}}, 14.88 \%$ less than $\overline{E_{\text {cr.1 }}^{a 23}}$ and $11.31 \%$ less than $\overline{E_{\text {cr.1 }}^{a 33}}$, reaching the goals that decrease the energy consumption of EV.

\section{Conclusion}

To search for the relationship between electric energy consumption and driving cycles, control methods of electric energy, a certain EV and electric energy consumption testing system were selected for the experiment, in NEDC and Jap1015 driving cycles. The relationship between electric energy consumption and driving cycles, control methods of electric energy was analyzed. The result shows that the electric energy consumption of EV do not increase as the driving distance is added in different driving cycles, the electric energy consumption of the entire driving cycle depends on the accelerating stage, and the dynamic control method of electric energy is the main influencing factor of electric energy consumption. When the three accelerating stages of NEDC former part is controlled with dynamic control method based on instantaneous energy consumption per kilometer, the energy consumption per kilometer can decrease effectively.

\section{Acknowledgement}

This study is sponsored by the special fund for construction of public welfare research and ability in Guangdong Province, P.R China under project titled "The prediction and control technology of cycle life of lithium iron phosphate power battery based on driving conditions" (Project No 2014B010106004). 


\section{References}

[1] Guo Konghui, Jiang Hui, Zhang Jianwei. Power-train matching and optimization of electric vehicles[J]Science Technology and Engineering, 2010, 10(16): 3892-3896.

[2] Chen Ming, Guo Lixin. Parameters Sensitivity Analysis of Dynamic Performance and Energy Consumption Economy of Battery Electric Vehicle[J]. Journal of Northeastern University(Natural Science) 2012, (05): 723-726.

[3] Younes Z, Boudet L, Suard F, et al. Analysis of the main factors influencing the energy consumption of electric vehicles[C] Electric Machines \& Drives Conference. IEEE, 2013: 247-253.

[4] Wang Zhenpo, Yao Limin, Shun Fengchun. Preliminary Study on Evaluation System of EV Energy Consumption Economy[J]. Transaction of Beijing Institute of Technology, 2005, (06): 479-482.

[5] Huang Wanyou, Cheng yong, Ji Shaobo, et al. The Effect of Driving Manners on EV Energy Consumption[J]. Transaction of Beijing Institute of Technology, 2013 (02): 144-150.

[6] Peng Yonglun, Wan Shanshan, Hu Fangfang, et al. Study on Electric Vehicle Driving Range and Energy Consumption Rate Test [J]. Standard Science, 2015, (03): 18-19.

[7] Karbowski D, Pagerit S, Calkins A. Energy consumption prediction of a vehicle along a user-specified real-world trip [C]. EVS26 International Battery, Hybrid and Fuel Cell Electric Vehicle Symposium, 2012, Los Angeles, USA: 1 - 12. 